\title{
Ortholog-based protein-protein interaction prediction and its application to inter-species interactions Sheng-An Lee ${ }^{\dagger 1,4}$, Cheng-hsiung Chan ${ }^{\dagger 1}$, Chi-Hung Tsai ${ }^{5}$, Jin-Mei Lai ${ }^{6}$, Feng- Sheng Wang7, Cheng-Yan Kao*4,5 and Chi-Ying F Huang*1,2,3,4
}

\author{
Address: ${ }^{1}$ Institute of Clinical Medicine, National Yang-Ming University, Taipei 112, Taiwan, ${ }^{2}$ Institute of Bio-Pharmaceutical Sciences, National \\ Yang-Ming University, Taipei 112, Taiwan, ${ }^{3}$ Institute of Biotechnology in Medicine, National Yang-Ming University, Taipei 112, Taiwan, \\ ${ }^{4}$ Department of Computer Science and Information Engineering, National Taiwan University, Taipei 10617, Taiwan, ${ }^{5}$ Institute for Information \\ Industry, Taipei, Taiwan, ${ }^{6}$ Department of Life Science, Fu-Jen Catholic University, Taipei Hsien 242, Taiwan and ${ }^{7}$ Department of Chemical \\ Engineering, National Chung Cheng University, Chia-Yi 621, Taiwan \\ Email: Sheng-An Lee - d93922005@ntu.edu.tw; Cheng-hsiung Chan - frankch@ntu.edu.tw; Chi-Hung Tsai - brick@iii.org.tw; Jin- \\ Mei Lai - bio2028@mails.fju.edu.tw; Feng-Sheng Wang - chmfsw@ccunix.ccu.edu.tw; Cheng-Yan Kao* - cykao@csie.ntu.edu.tw; Chi- \\ Ying F Huang* - cyhuang5@ym.edu.tw \\ * Corresponding authors †Equal contributors
}

from Asia Pacific Bioinformatics Network (APBioNet) Seventh International Conference on Bioinformatics (InCoB2008)

Taipei, Taiwan. 20-23 October 2008

Published: 12 December 2008

BMC Bioinformatics 2008, 9(Suppl I2):SI I doi:I0.II86/I47I-2105-9-SI2-SI I

This article is available from: http://www.biomedcentral.com/I47I-2I05/9/SI2/SII

(C) 2008 Lee et al; licensee BioMed Central Ltd.

This is an open access article distributed under the terms of the Creative Commons Attribution License (http://creativecommons.org/licenses/by/2.0),

which permits unrestricted use, distribution, and reproduction in any medium, provided the original work is properly cited.

\begin{abstract}
Background: The rapid growth of protein-protein interaction (PPI) data has led to the emergence of PPI network analysis. Despite advances in high-throughput techniques, the interactomes of several model organisms are still far from complete. Therefore, it is desirable to expand these interactomes with ortholog-based and other methods.

Results: Orthologous pairs of 18 eukaryotic species were expanded and merged with experimental PPI datasets. The contributions of interologs from each species were evaluated. The expanded orthologous pairs enable the inference of interologs for various species. For example, more than 32,000 human interactions can be predicted. The same dataset has also been applied to the prediction of host-pathogen interactions. PPIs between $P$. falciparum calmodulin and several $H$. sapiens proteins are predicted, and these interactions may contribute to the maintenance of host cell $\mathrm{Ca}^{2+}$ concentration. Using comparisons with Bayesian and structure-based approaches, interactions between putative HSP40 homologs of $P$. falciparum and the $H$. sapiens TNF receptor associated factor family are revealed, suggesting a role for these interactions in the interference of the human immune response to $P$. falciparum.

Conclusion: The PPI datasets are available from POINT http://point.bioinformatics.tw/ and POINeT http://poinet.bioinformatics.tw/. Further development of methods to predict hostpathogen interactions should incorporate multiple approaches in order to improve sensitivity, and should facilitate the identification of targets for drug discovery and design.
\end{abstract}




\section{Background}

Many genome-wide high throughput yeast two-hybrid analyses have generated PPI datasets for various model organisms. Moreover, systematic manual curation of human protein interactomes, including BioGRID [1], MIPS [2], IntAct [3], PINdb [4], DIP [5], HPRD [6] and MINT [7], has also generated significant, but far from complete, datasets. Therefore, in addition to an empirical screening of the interacting proteins of a given target, a comparative strategy should further facilitate functional annotation of uncharacterized proteins.

Using our knowledge of conserved interactions in other organisms (or interologs) [8] to elucidate the interacting networks of a particular target protein, we have previously established a publicly accessible and functional database, POINT (the Prediction Of INTeractome database) http:// point.bioinformatics.tw/[9]. The application of a similar concept and the addition of further filtering criteria have recently been reported and, as a result, have produced many outstanding studies such as Ulysses [10], OPHID [11] and HomoMINT [12]. Recently, additional highthroughput yeast two-hybrid experiments have generated an enormous number of human PPIs $[13,14]$, which now require assessments of their accuracy [15] and further evaluations using the concept of interologs. Conversely, interologs may be used to estimate the reliability of high throughput observations.

It is expected that the interactions between conserved orthologs, which are conserved genes and gene products in different species, will be conserved as well. However, accurate human interolog predictions inferred from different species are much less abundant than expected $[6,12]$. Additionally, some argue that interologs are less conserved than orthologs [12]. The extent to which ortholog-based PPI predictions can be applied has not been extensively analyzed.

In this work, orthologous pairs from 18 eukaryotic species have been expanded. Using experimental PPIs, interologs for these 18 species can be predicted and analyzed. This concept has been applied to host-pathogen PPI predictions. An analysis of predicted H. sapiens-P. falciparum interactions revealed PPIs that are highly related to the maintenance of $\mathrm{Ca}^{2+}$ levels in host cells. When comparing this method to other prediction methods, we find that this approach can complement Bayesian statistical methods [16] and structure-based methods [17].

\section{Results and discussion Orthologs shared by $\mathrm{H}$. sapiens and other model organisms}

The complete ortholog matrix from 18 eukaryotic species is shown in Additional File 1: Table S1. For brevity, only the orthologs between $H$. sapiens and five common model organisms are presented (Table 1). These orthologs were based on the HomoloGene database. Interologs were determined from the model organisms $M$. musculus (mouse), R. norvegicus (rat), D. melanogaster (fruit fly), C. elegans (worm) and S. cerevisiae (yeast).

Based on ortholog information, the conservation of genes and ortholog groups among 18 eukaryotic species were identified. We found 81 genes that were conserved in all 18 species presented in HomoloGene (Additional File 2: Table S2), suggesting that these genes are fundamental and/or vital to eukaryotes. Interestingly, 243 genes are missing in $P$. falciparum, but found in the other 17 species, including members of the proteosome, various ATP syn-

Table I: Numbers of ortholog shared by human and five model organisms

\begin{tabular}{|c|c|c|c|c|c|c|c|}
\hline \multirow{2}{*}{$\begin{array}{l}\text { Species } \\
(\text { Taxonomy ID) }\end{array}$} & \multirow{2}{*}{$\begin{array}{l}\text { Number of } \\
\text { Genes with } \\
\text { Orthologs }\end{array}$} & \multicolumn{6}{|c|}{ Number of Shared Orthologs Groups ${ }^{b}$} \\
\hline & & H. sapiens & M. musculus & R. norvegicus & D. melanogaster & C. elegans & S. cerevisiae \\
\hline H. sapiens (9606) & 19491 & 19491 (100\%) & $\begin{array}{c}16330 \\
(83.78 \%)\end{array}$ & $\begin{array}{c}15116 \\
(77.55 \%)\end{array}$ & $5039(25.82 \%)$ & $395 I(20.27 \%)$ & $1593(8.17 \%)$ \\
\hline $\begin{array}{l}\text { M. musculus } \\
(10090)\end{array}$ & 19142 & $\begin{array}{c}16330 \\
(85.31 \%)\end{array}$ & 19142 (100\%) & $\begin{array}{c}16674 \\
(87.11 \%)\end{array}$ & $4990(26.07 \%)$ & $3942(20.59 \%)$ & 1607 (8.39\%) \\
\hline $\begin{array}{l}\text { R. norvegicus } \\
(10116)\end{array}$ & 17766 & $\begin{array}{c}15116 \\
(85.08 \%)\end{array}$ & $\begin{array}{c}16674 \\
(93.85 \%)\end{array}$ & $17766(100 \%)$ & $4662(26.24 \%)$ & 3711 (20.89\%) & 1509 (8.49\%) \\
\hline $\begin{array}{l}\text { D. melanogaster } \\
\text { (7227) }\end{array}$ & 7794 & $5039(64.65 \%)$ & $4990(64.02 \%)$ & $4662(59.82 \%)$ & 7794 (100\%) & $3377(43.33 \%)$ & $1344(17.24 \%)$ \\
\hline C. elegans (6239) & 4971 & $395 \mathrm{I}(79.48 \%)$ & $3942(79.30 \%)$ & $3711(74.65)$ & 3377 (67.93\%) & 4971 (100\%) & $1189(23.92 \%)$ \\
\hline $\begin{array}{l}\text { S. cerevisiae } \\
(4932)\end{array}$ & 4589 & 1593 (34.7I\%) & 1607 (35.01\%) & $1509(32.88 \%)$ & I 344 (29.29\%) & II 89 (25.91\%) & $4589(100 \%)$ \\
\hline
\end{tabular}

aThese species are ranked by the number of genes with ortholog information available.

bThe percentage below the number of ortholog refers to the coverage on the species given in the left most column. 
thases and many mitochondria-related genes. While most species in the HomoloGene database share a high proportion of orthologs with other species (ranging from $48.3 \%$ in O. sativa to $87.4 \%$ in $H$. sapiens), less than $20 \%$ of the 5,266 genes in $P$. falciparum can be grouped with genes from other species. This suggests that the lifestyle and biological processes of this parasite deviate from those of other organisms.

\section{PPIs in the POINT database}

PPIs from the various model organisms were used to infer PPIs (interologs) in higher order organisms such as $H$. sapiens. Because experimental PPIs from the target organisms are needed to verify these inferred PPIs, collections of PPIs are essential for an ortholog-based approach. The POINT database has collected most of the available public PPI data for a range of organisms (Table 2). It contains more than 44,000 human PPIs with available ortholog information. In addition, more than 70,000 yeast interactions are available, suggesting that a considerable number of human interologs can be inferred. Most of these interactions were obtained from high-throughput techniques such as yeast two-hybrid screening, which is prone to a high rate of false positives and other errors. Within the high-confidence dataset, where only PPIs verified by two or more methods or reported in the literature two or more times are included, there are 28,559 human PPIs and 25,612 yeast PPIs with available ortholog information.

While the use of high-confidence PPIs eliminates many potential PPIs that are present in the available datasets, this trimming process reduces the false positive rate. Among the organisms listed in Table 2, S. cerevisiae shows the most dramatic drop in the number of PPIs when only high-confidence PPIs are selected. The reason for this is obvious: this species is a single cell organism. Most of the PPI datasets were obtained using high-throughput approaches, and have not been verified by other methods or reported independently in the literature. For H. sapiens, the number of high-confidence PPIs exceeds even those of yeast. However, some species in the HomoloGene database do not have PPI data available. For example, P. troglodytes (Chimpanzee) and C. familiaris (dog) have no inferred human interologs despite the large number of orthologs they share with $H$. sapiens.

\section{Interologs inferred from ortholog pairs}

Given $n$ objects in an undirected network (graph), there will be $n(n-1) / 2$ relationships among these $n$ objects and $\mathrm{n}^{*} \mathrm{n}$ relationships for a directed network. Since there are 19,491 human ortholog groups (Table 1), we therefore can assume that there are $19,491 *(19,491-1) / 2$ pairwise interactions among these gene products. Certainly, a complete graph is not reasonable or biologically feasible. However, we can assume that each interaction can be associated with a probability and that the probability for a non-interacting pair will be 0 . At this stage, we do not have enough information to assign a probability for each theoretical interaction. However, we can expand all $189,939,795$ interactions among these 19,491 orthologous groups.

The interologs were inferred from ortholog information. Using the orthologous groups shared by humans and other species, we can obtain the maximum number of interologs from currently available interactomes. Only two orthologous groups shared by more than two species can be used to infer interologs. For example, if orthologous group A is shared by humans and mice, and orthologous group B is also shared by humans and mice, there

Table 2: Protein-protein interactions collected in the POINT database.

\begin{tabular}{|c|c|c|c|c|}
\hline \multirow[t]{2}{*}{ Species (Taxonomy ID)a } & \multicolumn{2}{|c|}{ All Available PPIs } & \multicolumn{2}{|c|}{ Confident PPIs } \\
\hline & PPI & Orthologs Groups PPIb & PPI & Orthologs Groups PPI \\
\hline S. cerevisiae (4932) & 82445 & 70264 & 31162 & 25612 \\
\hline H. sapiens (9606) & 45378 & 44251 & 29074 & 28559 \\
\hline D. melanogaster (7227) & 29342 & $1407 \mid$ & 1106 & 764 \\
\hline C. elegans (6239) & 5267 & 1572 & 692 & 288 \\
\hline M. musculus (10090) & 3851 & 3746 & 1320 & 1291 \\
\hline P. falciparum (36329) & 2844 & 188 & 8 & 8 \\
\hline R. norvegicus ( 10116$)$ & 1469 & 1399 & 1003 & 964 \\
\hline A. thaliana (3702) & 1420 & 691 & 353 & 223 \\
\hline S. pombe (2848I2) & 356 & 227 & 163 & 98 \\
\hline G. gallus (903I) & 43 & $4 I$ & 17 & 16 \\
\hline O. sativa (39947) & 49 & 33 & 1 & I \\
\hline C. familiaris (96|5) & 2 & 2 & I & I \\
\hline
\end{tabular}

a These species are ranked by the number of available PPIs, except for Others and Inter-species.

b Orthologous Group PPIs are PPIs with ortholog information available. 
will be a potential interolog A-B between humans and mice, although the probabilities associated with these two interactions (one in human and one in mouse) are not known.

Based on this assumption, we analyzed a number of orthologous group pairs and identified a number of species sharing these orthologous groups for $\mathrm{H}$. sapiens (Additional File 3: Table S3). Among the 189,939,795 interactions, 180,191,177 interologs were inferred from ortholog information. This translates to $94.86 \%$ coverage of interologs $\left(I C^{H S A}\right)$. Although the theoretical interolog coverage is high (nearly 95\%), the interolog coverage on currently available PPIs is not significant. For all available human PPIs, only 3,859/44,251 interactions (8.72\%) can be inferred from known interactions in other model organisms. Using the trimmed set of high-confidence PPIs, this coverage drops to $4.61 \%(1,316 / 28,559)$. There is an obvious gap between the theoretical upper boundary and the experimentally observed data.

To investigate the origin of this gap, we further analyzed the interolog coverage of each model organism. Five common model organisms were selected. The number of inferable interologs, experimental PPI derived interologs and their interolog coverage were calculated (Table 3 and Table S3). It is interesting that the most commonly used model organism, S. cerevisiae (yeast), has a theoretical interolog coverage of only $0.67 \%$ (interologs inferred from yeast divided by all human interactions), whereas the ICHSA of M. musculus (mouse) and R. norvegicus (rat) are larger by two-orders of magnitude. However, for experimental human PPIs, the IC ${ }^{H S A}$ of mouse is only 2fold higher than that of yeast, and the $I C^{H S A}$ of rat is lower than that of yeast. The species contributions, $C^{S p}$, shown in this table are also informative. While mouse contributes $43.07 \%$ of the known interologs, yeast contributes only $19.85 \%$. This trend was mostly unchanged for highconfidence PPIs, except the contribution of yeast was boosted to $32.29 \%$.
The mapping of all orthologous group pairs permits interolog prediction for various eukaryotic species. For example, in the POINeT web service http:// poinet.bioinformatics.tw/, interologs can be inferred for seven eukaryotic species (H. sapiens, M. musculus, D. melangaster, C. elegans, S. cerevisiae, A. thaliana, and P. falciparum). Currently, more than 32,000 human interologs can be inferred. Among them, 3,859 have been confirmed by experimental evidence. The continual growth of interactomes in every eukaryotic species will continue to improve the ability to predict interologs.

\section{Prediction of inter-species host-pathogen interactions}

$P$. falciparum is a parasite with a complex life cycle, and this malarial parasite threatens millions of lives worldwide. Based on the HomoloGene database, P. falciparum has the least similar genome in comparison to other species. Only roughly $20 \%(990 / 5,266)$ of the genes share orthologous groups with other organisms. This suggests that many cellular processes vital to other eukaryotes may be missing or replaced in $P$. falciparum, and the interplay between the parasite and its two hosts may compensate for the functions missing in the $P$. falciparum genome. The interactome of $\mathrm{P}$. falciparum has been determined experimentally [18] and modeled genome-wide [19]. This allows comparisons to be done between the genomes and interactomes of $P$. falciparum and its two hosts, $H$. sapiens and A. gambiae (the African malaria mosquito).

Using the experimental PPIs and interologs, 3,090 interspecies interactions between $P$. falciparum and $H$. sapiens (and not intra-P. falciparum interactions) were found (Additional File 4: Table S4). The Gene Ontology annotations of the $P$. falciparum and $H$. sapiens genes were identified. These inter-species PPIs have been grouped based on the ontology of their biological processes. The resulting network is illustrated in Figure 1. The nodes in Figure 1 are biological processes from $P$. falciparum and H. sapiens. Links between $P$. falciparum and $H$. sapiens biological processes were derived from interactions linking two genes

Table 3: Contributions of model organisms to human theoretical and experimental interologs.

\begin{tabular}{|c|c|c|c|c|c|c|c|c|c|}
\hline \multirow[t]{3}{*}{ Species (Taxonomy ID) } & \multicolumn{3}{|c|}{ Theoretical Interologs Coverage ${ }^{a}$} & \multicolumn{6}{|c|}{ Experimental PPIs and Interologs ${ }^{b}$} \\
\hline & \multirow{2}{*}{$\begin{array}{l}\text { HomoloGene } \\
\text { ICHSA }\end{array}$} & \multirow{2}{*}{$\begin{array}{l}\text { OrthoMCL } \\
\text { ICHSA }\end{array}$} & \multirow{2}{*}{$\begin{array}{l}\text { TIGR EGO } \\
\text { ICHSA }\end{array}$} & \multicolumn{3}{|c|}{ All Available PPIs } & \multicolumn{3}{|c|}{ Confident PPIs } \\
\hline & & & & Interologs & $I C H S A$ & $C S P$ & Interologs & ICHSA & $C S P$ \\
\hline H. sapiens (9606) & $94.86 \%$ & $100.00 \%$ & $95.78 \%$ & 3859 & $8.72 \%$ & $\mathrm{~N} / \mathrm{A}$ & 1316 & $4.61 \%$ & N/A \\
\hline M. musculus (10090) & $70.19 \%$ & $77.56 \%$ & $50.24 \%$ & 1662 & $3.76 \%$ & $43.07 \%$ & 551 & $1.93 \%$ & $41.86 \%$ \\
\hline R. norvegicus ( $10 \mid 16)$ & $60.14 \%$ & $71.59 \%$ & $35.83 \%$ & 480 & $1.08 \%$ & $12.44 \%$ & 251 & $0.88 \%$ & $19.07 \%$ \\
\hline D. melanogaster (7227) & $6.68 \%$ & $12.01 \%$ & $4.79 \%$ & 766 & $1.73 \%$ & $19.85 \%$ & 92 & $0.32 \%$ & $7.00 \%$ \\
\hline C. elegans (6239) & $4.11 \%$ & $8.05 \%$ & $2.78 \%$ & 231 & $0.52 \%$ & $5.99 \%$ & 29 & $0.10 \%$ & $2.20 \%$ \\
\hline S. cerevisiae (4932) & $0.67 \%$ & $2.02 \%$ & $0.77 \%$ & 766 & $1.73 \%$ & $19.85 \%$ & 425 & $1.49 \%$ & $32.29 \%$ \\
\hline
\end{tabular}

alCHSA for theoretical interologs are the number of interologs divided by all theoretical human PPIs derived from each ortholog databases. b ICHSA for all available and confident experimental interologs are the number of interologs divided by available and confident human PPIs. 


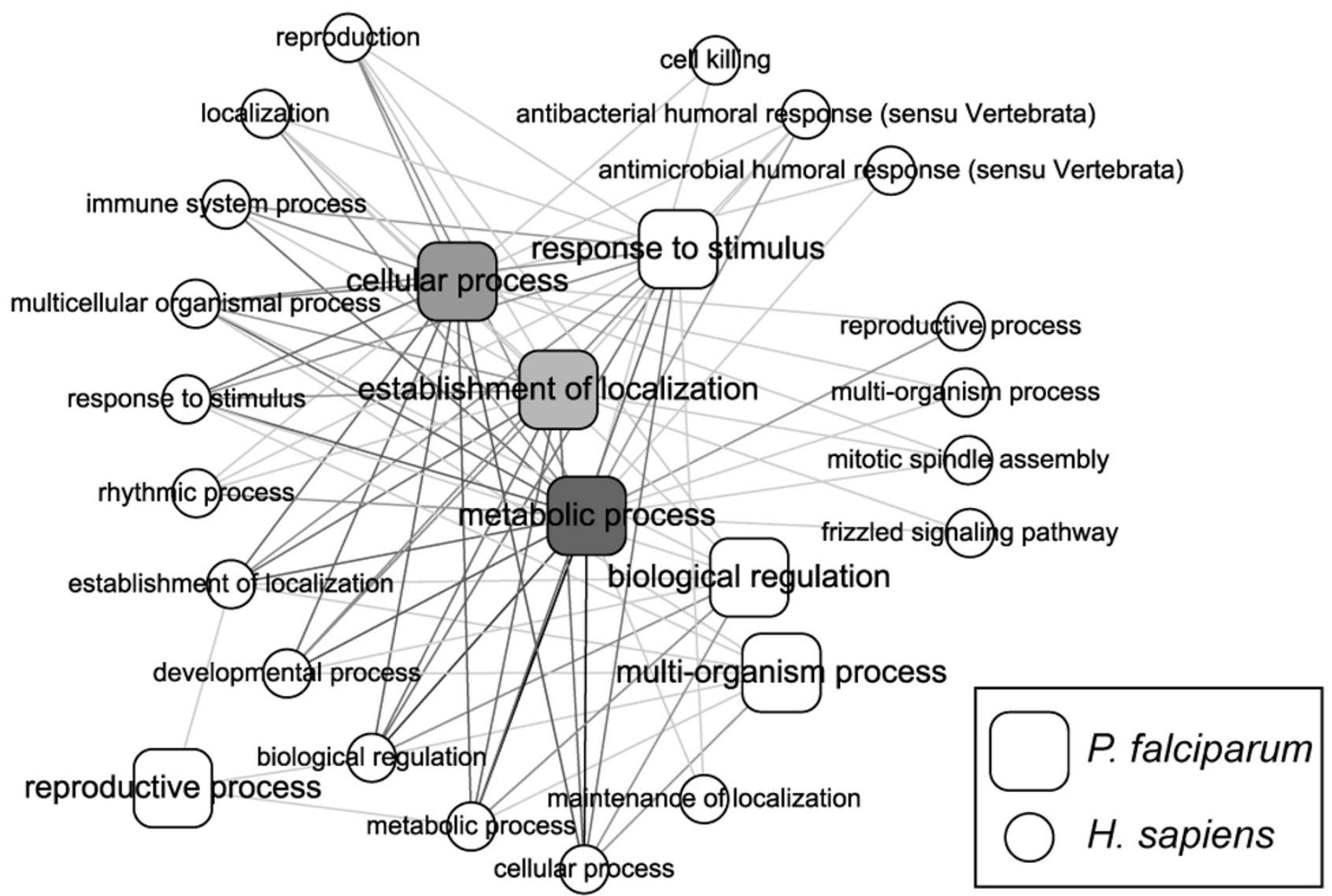

Figure I

Interactions between $P$. falciparum and $H$. sapiens are grouped by biological processes from Gene Ontology. Interactions between $P$. falciparum and $H$. sapiens are grouped by biological processes from Gene Ontology. Each node represents a GO biological process in either $P$. falciparum or $H$. sapiens. The nodes of biological processes for $P$. falciparum are shaded based on their involvement in the inter-species interaction network; darker color implies larger involvement. For $P$. falciparum, most of the interactions are related to metabolic and cellular processes.

that participate in the respective biological processes in the two species. Darker lines indicate the involvement of more interactions, allowing more interplay between the two biological processes. The $P$. falciparum biological processes are shaded using different levels of grey. Darker nodes indicate that more genes are involved in the process. In Figure 1, the metabolic processes and cellular processes of $P$. falciparum are most abundant in the hostparasite interaction network. This is understandable, since $P$. falciparum is a parasite and needs to acquire nutrients from the host erythrocyte. In the genomic-wide model of the $P$. falciparum interactome, only a small fraction of intra-P. falciparum interactions contributed to metabolic processes [19], which supports the notion that P. falciparum metabolic processeses may be dependant on human metabolic and cellular processes. There are also other interesting interactions between $P$. falciparum and the antimicrobial, antibacterial, cell killing and immune system processes of $H$. sapiens.

\section{Filtering and analysis of predicted inter-species interactions}

Although more than 3,000 H. sapiens-P. falciparum PPIs were inferred, not all of these interactions are likely to take place under physiological conditions due to spatiotemporal constraints. Filtering using gene ontology annotations resulted in 918 host-pathogen interactions. Further filtering of $P$. falciparum sequences using the presence/absence of translocational signals led to 95 PPIs (Figure 2). Only 15 P. falciparum proteins participate in these 95 PPIs (Table 4). One of the P. falciparum proteins, calmodulin (PF14_0323), interacts with 50 human proteins. It is well known that $P$. falciparum requires an environment with high $\mathrm{Ca}^{2+}$ levels [20], and the abundence of calmodulin- 


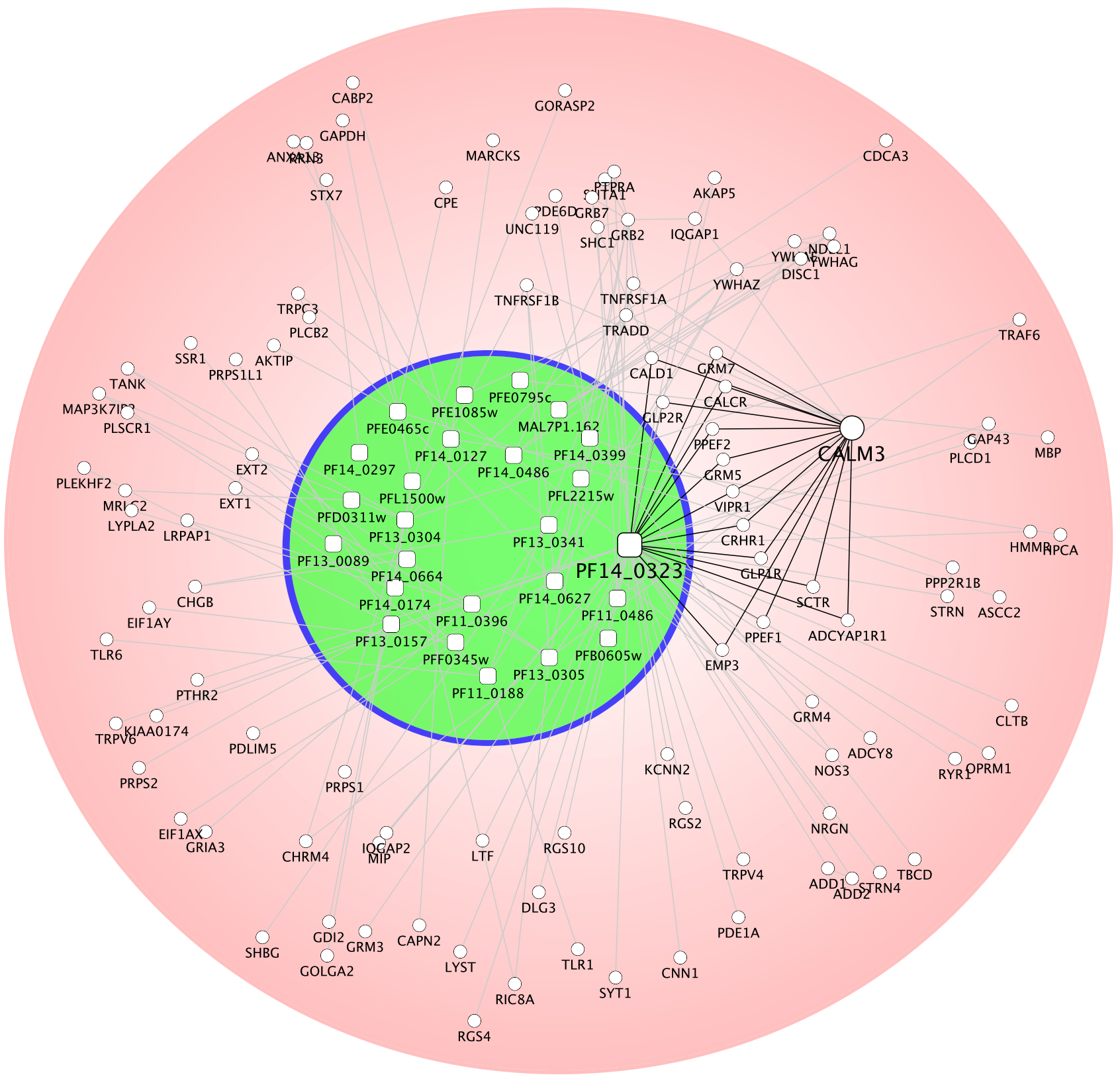

Figure 2

Illustration of filtered $\boldsymbol{H}$. sapiens-P. falciparum interactions. $P$. falciparum calmodulin (PFI4_0323) shares I3 interaction partners with human calmodulin (CALM3), suggesting competition between the two proteins, and interference of host cell $\mathrm{Ca}^{2+}$ homeostasis. (Red: red blood cell; Green: the parasitophorous vacuole).

based interactions may help $P$. falciparum maintain this high $\mathrm{Ca}^{2+}$ concentration [21]. Among the 50 human proteins interacting with PF14_0323, 13 also interact with human calmodulin (CALM3). This suggests that $P$. falciparum calmoduin shares some of the targets of human calmodulin, and may hijack these PPIs for its own purpose. The protein with the second highest number of interactions was N-myristoyltransferase (PF14_0127). Many proteins interacting with calmodulin require myristoylation in N-terminal [22-24], further supports the functioning of the calmodulin-centric network.

Previously, Dyer et al. [16] have inferred host-pathogen interactions using Bayesian statistics. H. sapiens-P. falciparum PPIs predicted by the Bayesian approach are mainly enriched in 'blood coagulation' and 'membrane integra- 
Table 4: P. falciparum proteins participate in 95 PPIs filtered from 918 host-pathogen interactions.

\begin{tabular}{lrlr}
\hline Gene Symbol & Gene ID & Description & Number of Interactions \\
\hline PFI4_0323 & 811905 & Calmodulin & 50 \\
PFI4_0I27 & 811708 & N-myristoyltransferase & 6 \\
PFI4_0627 & 812209 & ribosomal protein S3, putative & 6 \\
PFII_0I88 & 810735 & heat shock protein 90 & 6 \\
PFI4_0399 & $81198 I$ & ADP-ribosylation-like factor, putative & 4 \\
PFI3_0I57 & 814127 & ribose-phosphate pyrophosphokinase \\
MAL7PI.I62 & 2654986 & dynein heavy chain, putative & 4 \\
PFI4_0486 & 812068 & elongation factor 2 & 4 \\
PFII_0486 & 811029 & MAEBL \\
PFF0345w & 3885886 & translation initiation factor IF-2, putative \\
PFE0795c & 812973 & nif-like protein, putative & 3 \\
PFII_0396 & 810942 & Protein phosphatase 2C \\
PFB0605w & 812721 & Ser/Thr protein kinase, putative \\
PFI4_0664 & 812246 & biotin carboxylase subunit of acetyl CoA carboxylase, putative & 3 \\
PFI4_0297 & 811879 & ecto-nucleoside triphosphate diphosphohydrolase I, putative & 2 \\
& & 2 \\
\end{tabular}

tion' protein interactions. This may partly be due to the gene ontology terms used to filter the PPIs. It is difficult to compare the two works, since the datasets and methodology used are different. However, the intersection of the two datasets reveals 3 interactions between PF14_0359 and the TNF receptor associated factor family (TRAF1, TRAF2 and TRAF6). PF14_0359 is a hypothetical protein. Inspection of the HomoloGene database reveals that PF14_0359 may be a homolog of DNAJA1 (HSP40). The functional implications of these three interactions require further investigation. However, TNF associated factor family are known to be involved in host immune response, suggesting that $P$. falciparum may interfere with this defence mechanism in $H$. sapiens. All in all, the diversity of different host-pathogen interaction inference methods suggests that these and other approaches may complement each other. And further development of the ability to predict host-pathogen interactions may benefit from the combination of multiple diverse approaches.

\section{Conclusion}

The expansion of all orthologous pairs enables the inference of interologs for various eukaryotic organisms, as illustrated by POINeT http://poinet.bioinformatics.tw/. The same inference method can also be applied to the prediction of inter-species interaction, especially in the case of host-pathogen interactions. The H. sapiens-P. falciparum PPIs inferred in our work reveal that $P$. falciparum may utilize calcium modulating proteins in the host cell to maintain $\mathrm{Ca}^{2+}$ levels, and this may serve as a target for drug development strategies [25].

\section{Methods}

Ortholog information for interolog analysis

One of the limitations inherent in the analysis of interologs is the assignment of the orthologs, which is achieved using various BLAST algorithms together with several additional criteria $[6,9,11,26,27]$ or from the NCBI HomoloGene and other protein/gene cluster databases. In this work, the ortholog information for each human gene was identified using the NCBI HomoloGene Release 54 [28]. The NCBI HomoloGene database contains homologous information for 18 eukaryotic organisms and has been augmented with homology and phenotype information drawn from various sources, e.g., MGI [29] and Fly base [30].

\section{Collection of PPIs}

The new version of POINT integrated several publicly accessible PPI datasets (Additional File 5: Table S5). These data sources have diverse entry formats, disparate ID systems and different protein symbols. The diversity of these datasets made the task of performing cross-site browsing or iterative querying very tedious and challenging. We systematically re-organized these datasets to improve and standardize the publicly accessible PPIs. High-throughput PPI datasets are prone to false positives and errors. Therefore, we also generate a relatively high-confidence PPI subset, which refers to a PPI subset where the PPIs have been verified by two or more experimental methods or published in the literature two or more times.

\section{Evaluation of interolog coverage}

The interolog coverage is quantifiable from an estimation of the ortholog-based PPI prediction power. The definition of interolog coverage is as follows:

$$
I C^{H S A}=\frac{N}{T^{H S A}} \times 100 \%
$$

where $T^{H S A}$ is the total number of human (H. sapiens) interactions (whether theoretical, experimental, or highly confident), $N$ is the number of interologs, and $I C^{H S A}$ is the interolog coverage for the human interactome. Another 
measure is the contribution of a given model organism to the human interologs and this is defined as

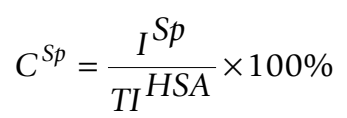

where $T I^{H S A}$ is the total number of human interologs, $I^{S p}$ is the number of interologs inferred from species $S p$, and $C^{S p}$ is the contribution of species $S p$ to human interologs.

\section{Inference and filtering of inter-species interactions}

With the expanded orthologous pairs, intra- and interspecies PPIs can be inferred with ease. The inference of $H$. sapiens-P. falciparum interactions are based on orthologous pairs with one-side orthology to $P$. falciparum. For example, given a PPI between $M_{a}$ and $M_{b}$ in species $M$, if $\mathrm{M}_{\mathrm{a}}$ has an ortholog in P. falciparum $\left(\mathrm{P}_{\mathrm{a}}\right)$, and $\mathrm{M}_{\mathrm{b}}$ has an ortholog $\mathrm{H}_{\mathrm{b}}$ in $H$. sapiens (but not in $P$. falciparum), an interaction between $\mathrm{P}_{\mathrm{a}}$ and $\mathrm{H}_{\mathrm{b}}$ is inferred.

However, interologs inferred from orthologous pairs may not occur in vivo, especially in the case of inter-species interactions. $P$. falciparum inhabits a parasitophorous vacuole after its entry into the red blood cell. A translocational signal peptide (RELXE/Q) is required to translocate $P$. falciparum proteins into red blood cell cytoplasm for host-cell manipulation [31-33]. Also, proteins localized in the nucleus (both H. sapiens and P. falciparum) are not likely to participate in inter-species PPIs. Two filters have been applied to reduce such unlikely cases. The first filter utilizes gene ontology annotations. Human proteins with the following annotations were removed: mitochondria, nucleus, ribosome, cell process, helicase activity, complex, nuclease activity, nucleic acid binding, nucleotide binding or proteolysis. The second filter utilizes the translocation signal RELXE/Q, where X refers to any amino acids. P. falciparum sequences matching this pattern within the first $25 \%$ of its length are kept, since translocation signals are likely to appear at the N-terminal.

\section{Competing interests}

The authors declare that they have no competing interests.

\section{Authors' contributions}

$\mathrm{CYH}, \mathrm{CYK}, \mathrm{FSW}$, and JML provide the concept and guidelines for the POINT/POINeT web servers. SAL collects and analyzes the protein-protein interaction and ortholog data, and predicts the inter-species interaction data. CHC proposes the inter-species and host-pathogen concept and writes the manuscript. CHT provides the literature about P. falciparum.

\section{Additional material}

\section{Additional file 1}

Table S1. The orthologous group coverage among 18 eukaryotic species. Click here for file

[http://www.biomedcentral.com/content/supplementary/1471-

2105-9-S12-S11-S1.xls]

\section{Additional file 2}

Table S2. Orthologous groups conserved in multiple species. Click here for file

[http://www.biomedcentral.com/content/supplementary/1471-

2105-9-S12-S11-S2.xls]

\section{Additional file 3}

Table S3. Interlog coverage and contributions for each species. Click here for file

[http://www.biomedcentral.com/content/supplementary/14712105-9-S12-S11-S3.xls]

\section{Additional file 4}

Table S4. Predicted inter-species interactions between P. falciparum and H. sapiens.

Click here for file

[http://www.biomedcentral.com/content/supplementary/14712105-9-S12-S11-S4.xls]

\section{Additional file 5}

Table S5. Protein-protein interaction data sources.

Click here for file

[http://www.biomedcentral.com/content/supplementary/14712105-9-S12-S11-S5.xls]

\section{Acknowledgements}

This research was supported by grants from the Program for Promoting Academic Excellence of Universities (National Yang Ming University) to $\mathrm{CYH}$ and the National Science Council (Program for Interdisciplinary Research Project: NSC95-2627-B-400-002 to CYH, NSC95-2627-B-030001 to JML, NSC96-2627-B-194-00 I to FSW, and NSC95-2627-B-002-0I I to CYK). This study was also partly funded by the "Gene Diagnostic Service Model Research and Niche Market Analysis" project of the Institute of Information Industry, supported by the Ministry of Economy Affairs of the Republic of China.

This article has been published as part of BMC Bioinformatics Volume 9 Supplement 12, 2008: Asia Pacific Bioinformatics Network (APBioNet) Seventh International Conference on Bioinformatics (InCoB2008). The full contents of the supplement are available online at http://www.biomedcentral.com/ |47|-2105/9?issue=S12.

\section{References}

I. Stark C, Breitkreutz BJ, Reguly T, Boucher L, Breitkreutz A, Tyers M: BioGRID: a general repository for interaction datasets. Nucleic Acids Res 2006, 34:D535-539.

2. Pagel P, Kovac S, Oesterheld M, Brauner B, Dunger-Kaltenbach I, Frishman G, Montrone C, Mark P, Stumpflen V, Mewes HW, et al: The MIPS mammalian protein-protein interaction database. Bioinformatics 2005, 21:832-834.

3. Hermjakob H, Montecchi-Palazzi L, Lewington C, Mudali S, Kerrien S, Orchard S, Vingron M, Roechert B, Roepstorff P, Valencia A, et al.: 
IntAct: an open source molecular interaction database. Nucleic Acids Res 2004, 32:D452-455.

4. Luc PV, Tempst P: PINdb: a database of nuclear protein complexes from human and yeast. Bioinformatics 2004, 20:14|3-14|5.

5. Salwinski L, Miller CS, Smith AJ, Pettit FK, Bowie JU, Eisenberg D: The Database of Interacting Proteins: 2004 update. Nucleic Acids Res 2004, 32:D449-45I.

6. Gandhi TK, Zhong J, Mathivanan S, Karthick L, Chandrika KN, Mohan SS, Sharma S, Pinkert S, Nagaraju S, Periaswamy B, et al.: Analysis of the human protein interactome and comparison with yeast, worm and fly interaction datasets. Nat Genet 2006, 38:285-293.

7. Zanzoni A, Montecchi-Palazzi L, Quondam M, Ausiello G, HelmerCitterich M, Cesareni G: MINT: a Molecular INTeraction database. FEBS Lett 2002, 5 I3:135-140.

8. Walhout AJ, Sordella R, Lu X, Hartley JL, Temple GF, Brasch MA, Thierry-Mieg N, Vidal M: Protein interaction mapping in C. elegans using proteins involved in vulval development. Science 2000, 287: $116-122$.

9. Huang TW, Tien AC, Huang WS, Lee YC, Peng CL, Tseng HH, Kao CY, Huang CY: POINT: a database for the prediction of protein-protein interactions based on the orthologous interactome. Bioinformatics 2004, 20:3273-3276.

10. Kemmer D, Huang Y, Shah SP, Lim J, Brumm J, Yuen MM, Ling J, Xu $\mathrm{T}$, Wasserman WW, Ouellette BF: Ulysses - an application for the projection of molecular interactions across species. Genome Biol 2005, 6:R I06.

II. Brown KR, Jurisica I: Online predicted human interaction database. Bioinformatics 2005, 21 :2076-2082.

12. Persico M, Ceol A, Gavrila C, Hoffmann R, Florio A, Cesareni G: HomoMINT: an inferred human network based on orthology mapping of protein interactions discovered in model organisms. BMC Bioinformatics 2005, 6(Suppl 4):S2I.

13. Stelzl U, Worm U, Lalowski M, Haenig C, Brembeck FH, Goehler H, Stroedicke M, Zenkner M, Schoenherr A, Koeppen S, et al:: A human protein-protein interaction network: a resource for annotating the proteome. Cell 2005, I 22:957-968.

14. Rual JF, Venkatesan K, Hao T, Hirozane-Kishikawa T, Dricot A, Li N, Berriz GF, Gibbons FD, Dreze M, Ayivi-Guedehoussou N, et al: Towards a proteome-scale map of the human protein-protein interaction network. Nature 2005, 437: $1173-1178$.

15. Deane CM, Salwinski L, Xenarios I, Eisenberg D: Protein interactions: two methods for assessment of the reliability of high throughput observations. Mol Cell Proteomics 2002, I:349-356.

16. Dyer MD, Murali TM, Sobral BW: Computational prediction of host-pathogen protein-protein interactions. Bioinformatics 2007, 23:і I59-166.

17. Davis FP, Barkan DT, Eswar N, McKerrow JH, Sali A: Host pathogen protein interactions predicted by comparative modeling. Protein Sci 2007, 16:2585-2596.

18. LaCount DJ, Vignali M, Chettier R, Phansalkar A, Bell R, Hesselberth JR, Schoenfeld LW, Ota I, Sahasrabudhe S, Kurschner C, et al.: A protein interaction network of the malaria parasite Plasmodium falciparum. Nature 2005, 438: 103-107.

19. Date SV, Stoeckert CJ Jr: Computational modeling of the Plasmodium falciparum interactome reveals protein function on a genome-wide scale. Genome Res 2006, 16:542-549.

20. Tromans A: Malaria: the calcium connection. Nature 2004, 429:253.

21. Gazarini ML, Thomas AP, Pozzan T, Garcia CR: Calcium signaling in a low calcium environment: how the intracellular malaria parasite solves the problem. / Cell Biol 2003, 16 I: 103-I I0.

22. Matsubara M, Titani K, Taniguchi H, Hayashi N: Direct involvement of protein myristoylation in myristoylated alanine-rich C kinase substrate (MARCKS)-calmodulin interaction. J Biol Chem 2003, 278:48898-48902.

23. Hayashi $N$, Nakagawa $C$, Ito $Y$, Takasaki $A$, Jinbo $Y$, Yamakawa $Y$, Titani K, Hashimoto K, Izumi Y, Matsushima N: Myristoylation-regulated direct interaction between calcium-bound calmodulin and $\mathbf{N}$-terminal region of pp60v-src. I Mol Biol 2004, 338:169-180.

24. Matsubara M, Jing T, Kawamura K, Shimojo N, Titani K, Hashimoto K, Hayashi N: Myristoyl moiety of HIV Nef is involved in regulation of the interaction with calmodulin in vivo. Protein Sci 2005 , I 4:494-503.
25. Scheibel LW, Colombani PM, Hess AD, Aikawa M, Atkinson CT, Milhous WK: Calcium and calmodulin antagonists inhibit human malaria parasites (Plasmodium falciparum): implications for drug design. Proc Natl Acad Sci USA 1987, 84:73 I0-73 I4.

26. Goffard N, Garcia V, Iragne F, Groppi A, de Daruvar A: IPPRED: server for proteins interactions inference. Bioinformatics 2003, 19:903-904.

27. von Mering C, Jensen LJ, Snel B, Hooper SD, Krupp M, Foglierini M, Jouffre N, Huynen MA, Bork P: STRING: known and predicted protein-protein associations, integrated and transferred across organisms. Nucleic Acids Res 2005, 33:D433-437.

28. Wheeler DL, Barrett T, Benson DA, Bryant SH, Canese K, Chetvernin V, Church DM, DiCuccio M, Edgar R, Federhen S, et al.: Database resources of the National Center for Biotechnology Information. Nucleic Acids Res 2007, 35:D5-I2.

29. Blake JA, Eppig JT, Bult CJ, Kadin JA, Richardson JE: The Mouse Genome Database (MGD): updates and enhancements. Nucleic Acids Res 2006, 34:D562-567.

30. Grumbling G, Strelets V: FlyBase: anatomical data, images and queries. Nucleic Acids Res 2006, 34:D484-488.

31. Hiller NL, Bhattacharjee S, van Ooij C, Liolios K, Harrison T, LopezEstrano C, Haldar K: A host-targeting signal in virulence proteins reveals a secretome in malarial infection. Science 2004, 306:1934-1937.

32. Marti M, Good RT, Rug M, Knuepfer E, Cowman AF: Targeting malaria virulence and remodeling proteins to the host erythrocyte. Science 2004, 306:1930-1933.

33. Przyborski J, Lanzer M: Parasitology. The malarial secretome. Science 2004, 306: 1897-1898.
Publish with Bio Med Central and every scientist can read your work free of charge

"BioMed Central will be the most significant development for disseminating the results of biomedical research in our lifetime. "

Sir Paul Nurse, Cancer Research UK

Your research papers will be:

- available free of charge to the entire biomedical community

- peer reviewed and published immediately upon acceptance

- cited in PubMed and archived on PubMed Central

- yours - you keep the copyright
BioMedcentral 